Revue québécoise de psychologie

québécoîse

de psychologie

\title{
ALLIANCE THÉRAPEUTIQUE ET INTERVENTION CONTRAINTE : RECENSION CRITIQUE DES PRATIQUES RECOMMANDÉES AUPRÈS D'ADOLESCENTS DE MINORITÉS ETHNOCULTURELLES THERAPEUTIC ALLIANCE AND MANDATORY INTERVENTIONS: CRITICAL REVIEW OF RECOMMENDED PRACTICES FOR ETHNOCULTURAL MINORITY ADOLESCENTS
}

\section{Gary Saint-Jean et Sarah Dufour}

Volume 39, numéro 1, 2018

LE MULTICULTURALISME

MULTICULTURALISM

URI : https://id.erudit.org/iderudit/1044843ar

DOI : https://doi.org/10.7202/1044843ar

Aller au sommaire du numéro

Éditeur(s)

Revue québécoise de psychologie

ISSN

2560-6530 (numérique)

Découvrir la revue

Citer cet article

Saint-Jean, G. \& Dufour, S. (2018). ALLIANCE THÉRAPEUTIQUE ET INTERVENTION CONTRAINTE : RECENSION CRITIQUE DES PRATIOUES RECOMMANDÉES AUPRÈS D'ADOLESCENTS DE MINORITÉS ETHNOCULTURELLES. Revue québécoise de psychologie, 39(1), 35-63. https://doi.org/10.7202/1044843ar

\section{Résumé de l'article}

Le but de cette recension est de faire le point sur les pratiques cliniques recommandées afin de favoriser l'alliance thérapeutique dans l'intervention auprès d'adolescents de minorités ethnoculturelles dans des contextes d'intervention contrainte (protection de la jeunesse, justice pénale pour adolescents, psychiatrie). L'analyse des résultats des 32 articles sélectionnés permet de répertorier des pratiques s'organisant autour du savoir, du savoir-être et du savoir-faire. Cette analyse met également en lumière des biais méthodologiques importants dans les recherches entourant le sujet et une absence de soutien empirique des pratiques. Des orientations pour la pratique clinique et les recherches futures sont proposées. 


\section{ALLIANCE THÉRAPEUTIQUE ET INTERVENTION CONTRAINTE : RECENSION CRITIQUE DES PRATIQUES RECOMMANDÉES AUPRÈS D'ADOLESCENTS DE MINORITÉS ETHNOCULTURELLES}

THERAPEUTIC ALLIANCE AND MANDATORY INTERVENTIONS: CRITICAL REVIEW OF RECOMMENDED PRACTICES FOR ETHNOCULTURAL MINORITY ADOLESCENTS

La disproportion des groupes de minorités ethnoculturelles dans le système de protection de la jeunesse est un enjeu qui prend de plus en plus d'importance auprès des chercheurs et des intervenants œuvrant avec cette clientèle (Lavergne, Dufour et Couture, 2014). Cette disproportion, impliquant une sous-représentation ou une surreprésentation, se définit comme étant un déséquilibre entre la proportion de certains groupes d'enfants issus de minorités ethnoculturelles au sein du système de protection de la jeunesse et le poids démographique de ces groupes dans la population générale (Lavergne et al., 2014). Le terme " ethnoculturel » réfère à un groupement humain possédant une structure familiale, économique et sociale homogène. Cet ensemble de personnes partage généralement la même culture ainsi que les mêmes traditions et coutumes, qui se transmettent de génération en génération. Le terme réfère également aux acquisitions sociales issues du milieu dans lequel vit ce groupement (Ministre de l'Industrie, 2013). Le statut de "minorité » désigne que ces groupes sont inférieurs en nombre relativement au groupe majoritaire dans un territoire donné. Au Canada et aux États-Unis, ces groupes sont en minorité par rapport à une population majoritairement caucasienne.

Aux États-Unis, la disproportion a été particulièrement bien documentée. Les études démontrent que les enfants noirs sont surreprésentés dans le système de protection de la jeunesse, et ce, à toutes les étapes de la trajectoire de services (Anyon, 2011; Drake et al., 2011). La disproportion est cependant particulièrement notable à l'entrée des services de protection (Hill, 2006) et à l'étape du placement, où ces enfants sont au moins deux fois plus susceptibles d'être retirés de leur milieu familial. Au Québec, ce groupe est surreprésenté à la fois dans le système judiciaire pour adolescents et dans le système de protection de la jeunesse (Eid, Turenne et Magloire, 2011). Les études indiquent que des conditions sociales et économiques semblent compter pour beaucoup dans cette disproportion (pauvreté, secteur de résidence, discrimination, etc.). Des biais culturels et des pratiques discriminatoires de la part des professionnels

1. Adresse de correspondance: Département de psychologie, Université de Montréal, C.P. 6128, succ. Centre-ville, Montréal (QC), H3C 3J7. Téléphone : 514-692-8036. Courriel : gary.saint-jean@umontreal.ca 
et des citoyens (Fluke, Harden, Jenkins et Ruehrdanz, 2011; Lavergne et al., 2014) et un manque de ressources culturellement adaptées viendraient également exacerber cette problématique (Lavergne et al., 2014).

La littérature entourant la disproportion des adolescents de minorités ethnoculturelles dans le système de protection de la jeunesse et dans le système judiciaire pour adolescents met en lumière que certaines variables individuelles pourraient également jouer un rôle dans la disproportion, notamment le stress lié au fait de devoir s'adapter à différents systèmes culturels et à différents systèmes de valeurs (Pineau-Villeneuve, 2015). L'adaptation à ses différents systèmes vient donc complexifier la tâche de construction de l'identité à l'adolescence pour ces jeunes, car ils sont confrontés à des défis supplémentaires sur le plan identitaire qui viennent influencer leur adaptation psychologique et sociale.

Un de ces défis, l'acculturation, réfère à la manière dont un individu s'intègre à la culture du pays d'accueil alors qu'il a une origine ou une appartenance ethnoculturelle différente. Plus spécifiquement, ce concept désigne à quel point la personne désire conserver son héritage culturel et les coutumes qui s'y rattachent, mais aussi à quel point cette même personne cherche à s'impliquer dans sa société d'accueil (Berry, 2005). Essentiellement, la stratégie d'acculturation d'un adolescent de minorité ethnoculturelle pourrait prédire son ajustement psychologique et social. En fait, la combinaison d'une forte association à la culture d'origine et à la culture d'accueil prédit une meilleure adaptation, alors qu'une faible association aux deux prédit une mauvaise adaptation. Le maintien de la culture d'origine semble toutefois jouer un rôle plus important dans l'adaptation que l'implication dans la culture d'accueil (Berry, Phinney, Sam et Vedder, 2006; Soriano, Rivera, Williams, Daley et Reznik, 2004).

Les jeunes de minorités ethnoculturelles sont également confrontés au défi de développer leur identité ethnique dans une culture d'accueil différente de leur culture d'origine (Phinney, 1989). L'identité ethnique se définit comme un construit à plusieurs facettes qui implique le concept de soi en regard aux connaissances qui découlent de son appartenance à un groupe culturel donné (Torres et Ong, 2010). Alors qu'ils accordent généralement peu d'importance à leur appartenance ethnique avant l'adolescence, ces jeunes prennent de plus en plus conscience de la discrimination raciale qui les entoure, les amenant ainsi à devoir prendre position sur leur identité ethnique et à acquérir les habiletés nécessaires pour faire face à la discrimination et aux préjugés (Gonzales, 2014; Phinney, 1989). La formation de l'identité ethnique est associée à un meilleur ajustement psychologique (Gonzales, 2014) et est influencée par une multitude de facteurs, comme l'utilisation de la langue de la culture d'origine, 
la fréquentation de pairs de ce même groupe et la promotion de la culture d'origine par les parents (Phinney, Romero, Nava et Huang, 2001).

À ces défis sur le plan identitaire s'ajoutent les conflits intergénérationnels entre adolescents et parents qui peuvent être influencés par plusieurs variables, dont l'identité ethnique, l'intégration au pays d'accueil et la maitrise de la langue et des normes du pays d'accueil (Hassan et Rousseau, 2007; Malhamé, 2010). Les différences au niveau du vécu d'immigration et d'acculturation peuvent également accentuer ces conflits (Berry, 2005; Malhamé, 2010).

Le caractère contraint des services d'interventions mandatées par la loi rend également difficiles l'engagement et la collaboration des familles qui reçoivent des services (Cahalane, 2013). Ceux-ci sont perçus comme étant intimidants et stressants (Dumbrill, 2006). La relation de pouvoir inhérente entre intervenants et receveurs de services vient diminuer les droits et la liberté de choix des familles, tout en permettant aux intervenants d'exercer un contrôle sur ces derniers (Gladstone et al., 2014; Lemay, 2013). La manière dont les intervenants exerceraient ce pouvoir dans les services d'intervention contrainte aurait toutefois une forte influence sur le niveau de collaboration des familles dans ce contexte où les interventions autoritaires auraient tendance à diminuer la collaboration, alors qu'une intervention en partenariat la favoriserait (Dumbrill, 2006).

Afin de réduire la disproportion de certains groupes de minorités ethnoculturelles dans les services de protection et de justice pénale, plusieurs auteurs suggèrent qu'il serait essentiel de former des intervenants culturellement compétents pour pallier les barrières de langues et de communication et permettre aux familles de minorités ethnoculturelles d'accéder à des services (Chand, 2000; Fluke et al., 2011; Hines, Lemon, Wyatt et Merdinger, 2004; Lavergne et al., 2014). II faudrait également travailler en partenariat avec ces familles à toutes les étapes du service (Fluke et al., 2011; Lavergne et al., 2014). Ceci renvoie au fait qu'il est essentiel d'obtenir la collaboration des familles auprès de qui l'intervention est conduite (Couture et Dufour, 2013). Toutefois, le fait que ces services soient plus souvent reçus sous la contrainte légale a comme effet de rendre difficiles l'engagement, la collaboration et l'établissement d'une alliance avec les familles qui reçoivent ces services perçus comme intimidants et stressants (Cahalane, 2013; Dumbrill, 2006).

La relation intervenant-client constitue le meilleur moteur de changement en contexte de protection (Dumbrill, 2010; Gladstone et al., 2014), pointant vers l'importance d'établir une bonne alliance thérapeutique auprès des adolescents de minorités ethnoculturelles et de leur famille. L'établissement et le maintien de cette alliance sont donc un levier important 
dans l'intervention auprès de cette clientèle afin de réduire la disproportion constatée dans la prestation des services d'intervention contrainte. Toutefois, aucune étude dans la littérature actuelle ne propose de recommandations explicites aux intervenants pour les aider à favoriser l'alliance auprès de ce groupe spécifique de population. Afin de faire le point sur l'ensemble des connaissances disponibles à ce jour, une recension critique a été effectuée pour répondre à la question suivante : quelles sont les pratiques cliniques recommandées pour favoriser l'établissement de l'alliance thérapeutique auprès d'adolescents de minorités ethnoculturelles en contexte d'intervention contrainte?

\section{MÉTHODOLOGIE}

Les articles recensés ont été sélectionnés à partir de recherches dans les bases de données suivantes: PsychINFO, Web of sciences, Social Work Abstracts, Social Services Abstracts, Criminal Justice Abstracts, Érudit, Google Scholar et Medline. La sélection des mots-clés pour les différents moteurs de recherche a été supervisée par un bibliothécaire spécialisé en sciences sociales. Les articles publiés en français ou en anglais entre 2001 et 2016 ont été inclus dans la recension afin de repérer les connaissances les plus récentes. Les articles scientifiques, les thèses et mémoires, les chapitres de livre et les rapports et documents pratiques ont été recensés, alors que les éditoriaux et les livres entiers ont été exclus. Tous les types de devis ont été retenus (théorique, qualitatif, recension systématique, étude de cas, corrélationnel, quasi expérimental et expérimental). Les études recensées incluent, de manière non exclusive, des adolescents âgés de 12 à 25 ans issus de minorités ethnoculturelles vivant dans un pays où la population est majoritairement blanche et recevant des services d'intervention contrainte (des services d'intervention mandatée par la loi) dans un des domaines suivants : protection de la jeunesse, justice pénale pour adolescents ou psychiatrie. La tranche d'âge a été choisie afin d'aller chercher le plus grand nombre d'études possibles. Étant donnée la variabilité des âges limites pour les mandats de prise en charge et la pertinence des recommandations les concernant, les jeunes adultes ont été inclus dans la recension. Bien que les interventions dans ces domaines soient régies par des lois qui contraignent les jeunes et les familles à recevoir de l'aide, ces services peuvent aussi être obtenus de façon volontaire. Dans le cadre de la recension, seules les études rapportant de manière explicite la nature volontaire des services reçus par leurs participants ont été exclues. Les recherches dont les tranches d'âge de la population à l'étude n'incluaient que 2 ans ou moins de la tranche d'âge visée ont également été exclues (par ex., étude sur les jeunes de 9 à 13 ans). Les études se rapportant exclusivement aux populations autochtones

ont été exclues, car le portrait des difficultés de ce groupe de population se 
différencie de celui des groupes de minorités ethnoculturelles issus de l'immigration.

Seules les recommandations cliniques visant directement l'action auprès des jeunes ou des acteurs pertinents afin de favoriser l'établissement et le maintien de l'alliance thérapeutique ont été recensées. Tous les termes connexes à l'alliance thérapeutique (engagement, collaboration, etc.) ont également été inclus dans la recension. Les recommandations visant des composantes structurelles et organisationnelles, telles que les politiques et la législation, ont été exclues afin d'obtenir exclusivement des recommandations applicables par les intervenants auprès des jeunes et de leur entourage. Les études de réplication d'interventions existantes auprès d'une population de minorités ethnoculturelles sans modifications ainsi que les interventions ciblant un groupe spécifique de minorités ethnoculturelles sans groupe contrôle ou comparaison avec le groupe ethnoculturel majoritaire ont également été exclues puisqu'elles ne permettent pas de formuler des recommandations pour l'intervention spécifique auprès de minorités ethnoculturelles.

Étant donné l'absence de résultats répondant directement à la question de recherche, elle a été divisée afin de recenser de manière individuelle trois axes de recherches complémentaires. Les critères d'inclusion et d'exclusion mentionnés ci-haut s'appliquent donc à chacune de ces "sousrecensions ", mais la notion d'alliance thérapeutique est exclue de la recension 1, la notion de minorités ethnoculturelles est exclue de la recension 2 et la notion d'intervention contrainte est exclue de la recension 3 (voir le Tableau 4 en appendice pour les combinaisons de mots-clés utilisées).

Recension 1 : Quelles sont les pratiques cliniques recommandées pour une intervention culturellement compétente auprès des adolescents de minorités ethnoculturelles en contexte d'intervention contrainte? Parmi les 336 articles repérés dans les bases de données, 300 ont été exclus à la suite de la lecture des résumés parce qu'ils ne répondaient pas aux critères d'inclusion. Des 36 articles lus et analysés, 24 articles ont été exclus, car ils ne répondaient pas au critère d'inclusion ou car aucune recommandation clinique répondant à la question de recherche n'était proposée. Au final, 12 articles ont donc été inclus dans cette recension (voir la Figure 1 pour le processus de sélection).

Recension 2 : Quelles sont les pratiques cliniques recommandées pour favoriser l'établissement et le maintien d'une alliance thérapeutique dans l'intervention auprès d'adolescents en contexte d'intervention contrainte? Parmi les 172 articles repérés dans les bases de données, 140 ont été 


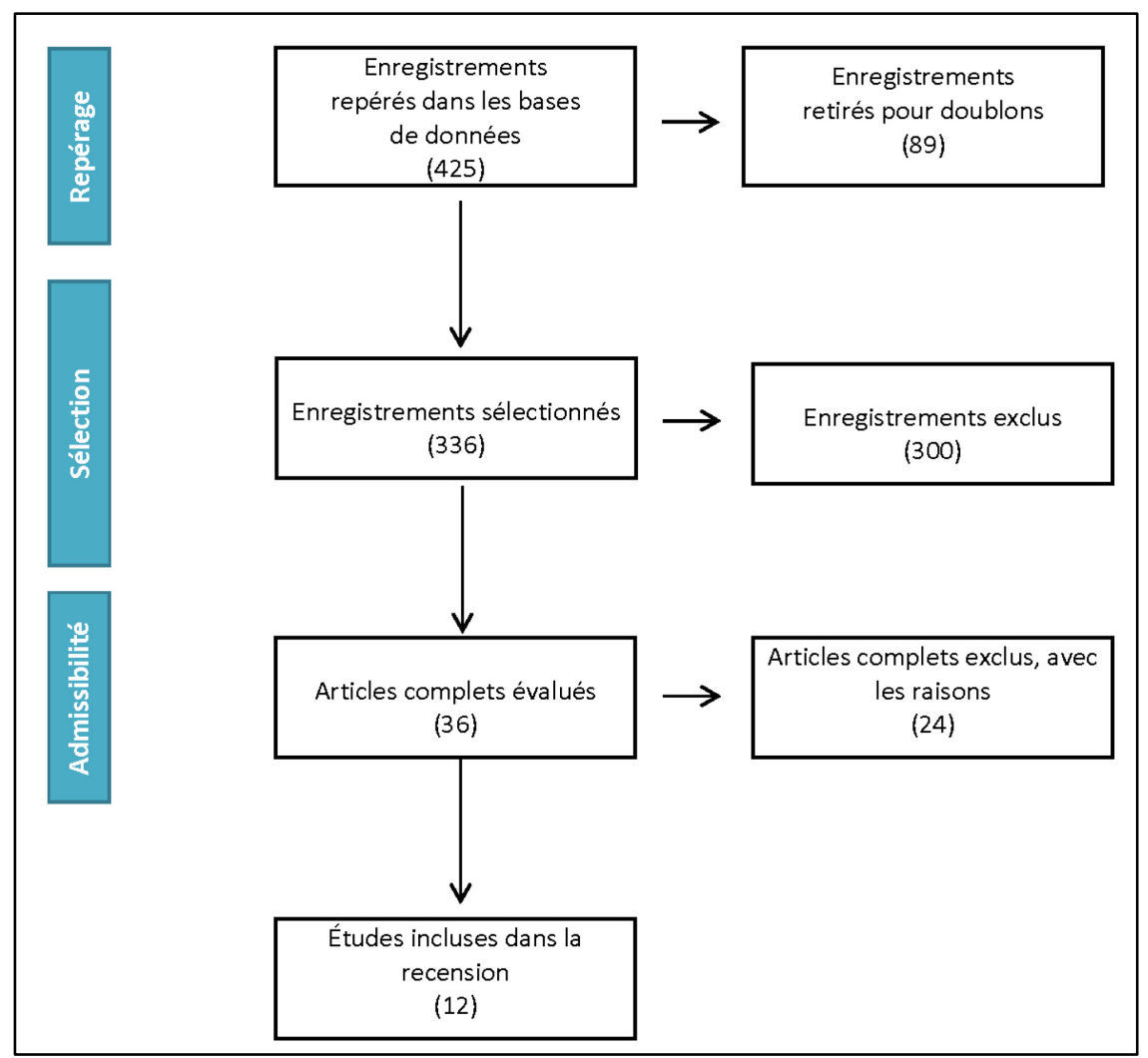

Figure 1 : Processus de sélection des articles de la recension 1

exclus à la suite de la lecture des résumés parce qu'ils ne répondaient pas aux critères d'inclusion. Après la lecture des 32 articles restants, 8 références tirées des articles ont été ajoutées étant donné leur pertinence potentielle. Des 40 articles lus et analysés, 31 articles ont été exclus, car ils ne répondaient pas au critère d'inclusion ou, car aucune recommandation clinique répondant à la question de recherche n'était proposée. Au final, 9 articles ont donc été inclus dans cette recension (voir la Figure 2 pour le processus de sélection).

Recension 3 : Quelles sont les pratiques cliniques recommandées pour une intervention culturellement compétente auprès d'adolescents de minorités ethnoculturelles qui favorise l'établissement et le maintien de l'alliance thérapeutique? Parmi les 145 articles repérés dans les bases de données, 121 ont été exclus à la suite de la lecture des résumés, car ils ne répondaient pas aux critères d'inclusion. Des 24 articles lus et analysés, 13 
RQP, 39(1)

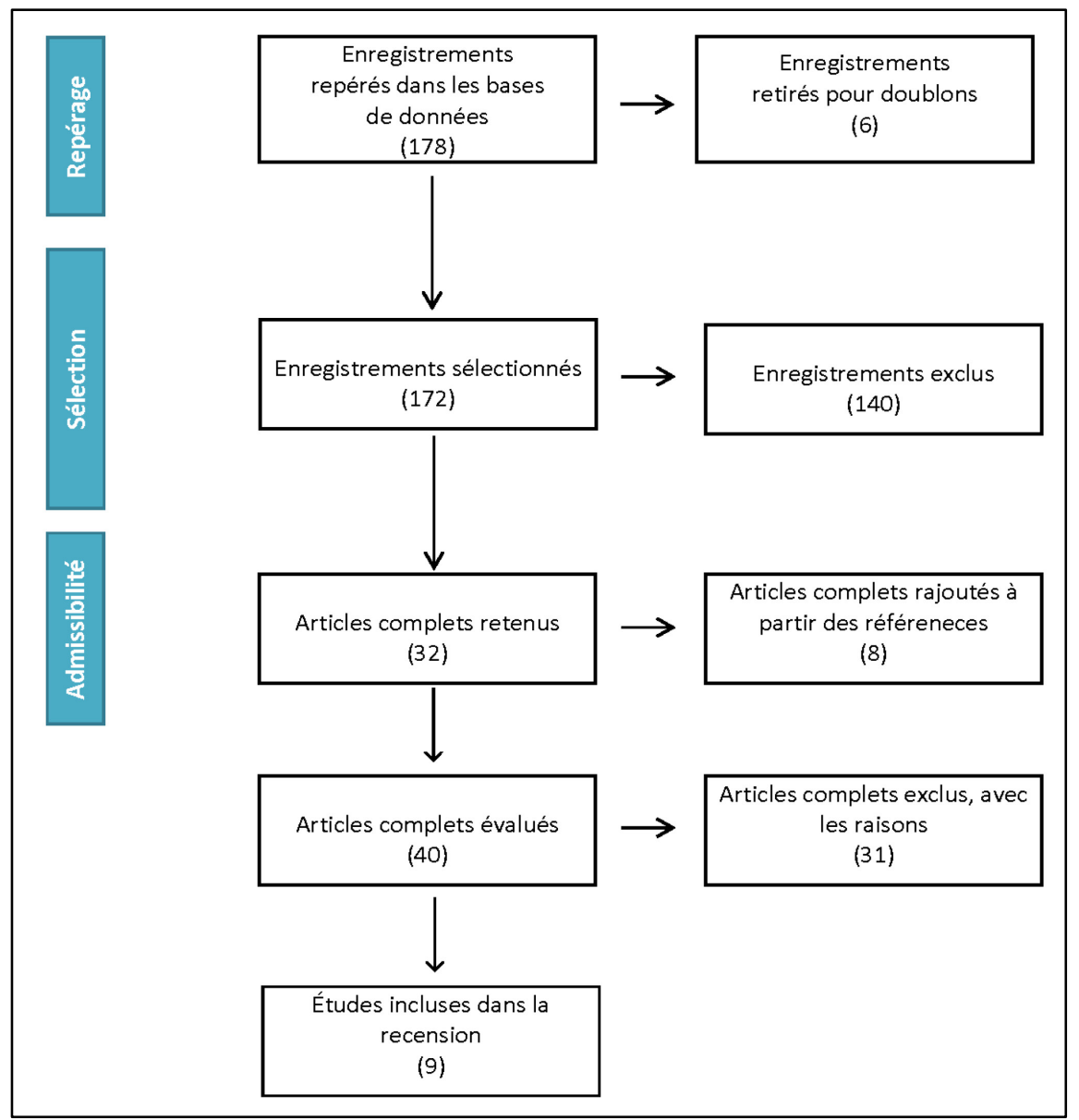

Figure 2 : Processus de sélection des articles de la recension 2

articles ont été exclus, car ils ne répondaient pas au critère d'inclusion ou car aucune recommandation clinique répondant à la question de recherche n'était proposée. Au final, 11 articles ont donc été inclus dans cette recension (voir la Figure 3 pour le processus de sélection).

Finalement, une analyse thématique a été effectuée avec les recommandations recensées dans les 32 articles sélectionnés afin de les regrouper dans des thèmes communs. Voir les Tableaux 1,2 et 3 pour les caractéristiques des articles recensés (à la fin de l'article). 


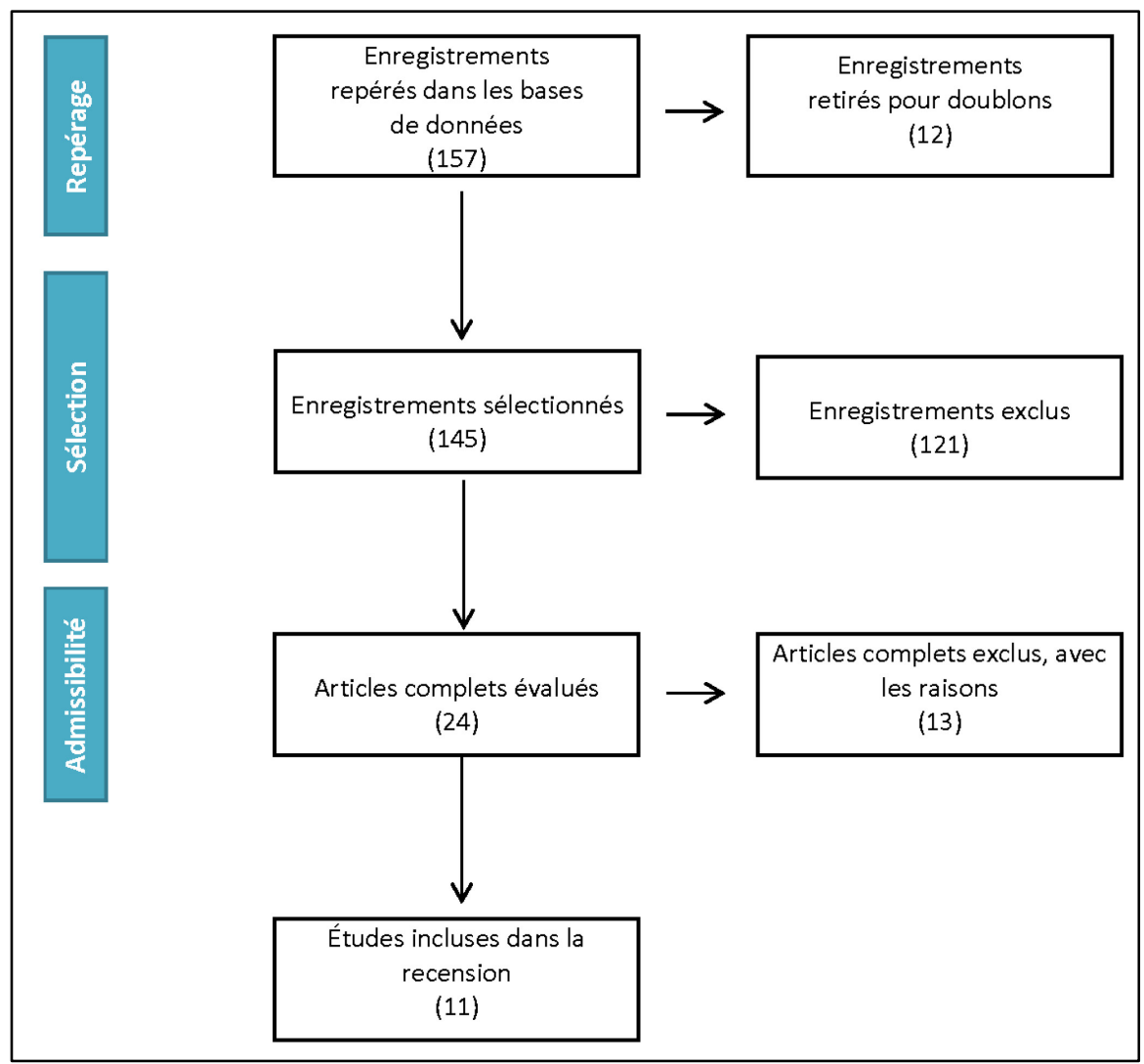

Figure 3 : Processus de sélection des articles de la recension 3

\section{RÉSULTATS}

Les recommandations cliniques recensées dans les articles analysés seront présentées selon trois domaines de compétences largement établis en relation d'aide: le savoir, le savoir-faire et le savoir-être. Le savoir désigne l'ensemble des connaissances théoriques de l'intervenant. Le savoir-faire désigne les compétences et habiletés procédurales maitrisées par l'intervenant, alors que le savoir-être désigne plutôt des attitudes véhiculées par l'intervenant (Aubret et Gilbert, 2003; Gendreau, 2001; Renou, 2005).

\section{Savoir}

Les savoirs critiques au regard de l'établissement et du maintien de l'alliance thérapeutique avec des adolescents de minorités ethnoculturelles 
recevant de l'aide contrainte sont de trois ordres: connaissances sur les plans culturel, linguistique et contextuel.

\section{Connaissances culturelles}

L'intervenant œuvrant auprès d'adolescents de minorités ethnoculturelles en contexte d'intervention contrainte doit, afin de favoriser l'alliance thérapeutique, détenir des connaissances préalables sur la culture de son client, notamment par rapport aux normes, croyances et valeurs propres à sa culture (Briggs et McBeath, 2010; Pumariega, Rothe, Song et Lu, 2010). L'intervenant doit aussi connaître le contexte communautaire dans lequel cette culture évolue (par exemple, le contexte de vie des Maghrébins aux États-Unis), ainsi que l'histoire de discrimination et les barrières systémiques rencontrées par ce groupe ethnoculturel dans le pays d'accueil (Gallardo et Curry, 2009; Lindsey, 2010; Venable et Guada, 2014). Les intervenants doivent également comprendre la conception de maladie mentale et de difficultés d'adaptation dans la culture du jeune (BrelandNoble, Burriss et Poole, 2010). Par exemple, un intervenant œuvrant auprès d'un adolescent afro-américain devrait savoir que certains membres de ce groupe de population considèrent difficilement la dépression comme un trouble clinique, voyant plutôt cette condition comme guérissable par la foi religieuse (Breland-Noble et al., 2010). De telles connaissances devraient être enseignées par le biais de formations à la compétence culturelle (Briggs et McBeath, 2010; Sharkey, Sander et Jimerson, 2010).

\section{Connaissances linguistiques}

Il est optimal pour l'intervenant de maîtriser la langue d'origine du jeune de minorités ethnoculturelles auprès duquel il intervient et de sa famille. Dans les nombreuses situations où ce n'est pas le cas, il est essentiel que l'intervenant soit accompagné par un interprète dûment formé ayant les connaissances nécessaires à la traduction linguistique et à l'interprétation du discours des receveurs de services (Pumariega et Rothe, 2003; Pumariega et al., 2013; Pumariega et al., 2010). Ces interprètes doivent être familiers avec la terminologie spécialisée utilisée par les donneurs de services (soit le vocabulaire propre à la psychiatrie, la protection de la jeunesse ou la justice pénale pour adolescents) et rendre compte tant du langage verbal que non-verbal des parties en présence (Pumariega et Rothe, 2003; Pumariega et al., 2010).

\section{Connaissances contextuelles}

Au-delà des connaissances propres à la culture d'origine du jeune et de sa famille, l'intervenant doit également comprendre les contextes social, politique et économique dans lesquels la famille et le jeune vivent (Briggs et McBeath, 2010). L'intervenant doit aussi être familier avec les normes et comportements valorisés dans le milieu de vie du jeune, tout en étant 
conscient de leurs éventuels changements. Ces normes ou comportements attendus, ancrés localement, peuvent être spécifiques à une région, un quartier ou une école (Sinclair et Smith, 2016). La connaissance de ces paramètres permet d'être conscient des influences possibles sur les comportements de l'adolescent et de l'intervenant ainsi que sur leur alliance. Par exemple, certains contextes peuvent accentuer les comportements de rébellion chez les jeunes et les comportements autoritaires chez les intervenants (Orsi, Lafortune et Brochu, 2010), nuisant ainsi à l'établissement d'une alliance thérapeutique entre eux.

\section{Savoir-faire}

Le domaine du savoir-faire se divise en deux sections. La première concerne exclusivement les compétences que l'intervenant doit exercer dans la relation dyadique avec l'adolescent de minorités ethnoculturelles en contexte d'intervention contrainte. La deuxième réfère aux compétences que l'intervenant doit manifester dans ses relations auprès des partenaires impliqués dans les différents milieux de vie de l'adolescent.

\section{Compétences dans la relation dyadique auprès de l'adolescent de minorités ethnoculturelles}

Les savoir-faire auprès du jeune s'organisent autour de la culture, de l'immigration, de la communication, des difficultés et symptômes et des buts et moyens.

Culture. La prise en compte de la culture constitue un élément central dans l'intervention auprès de toute clientèle de minorités ethnoculturelles afin de favoriser l'alliance thérapeutique, les adolescents en contexte d'intervention contrainte. II est donc recommandé de s'engager dans des interactions culturellement compétentes qui incorporent les valeurs, les coutumes, les croyances spirituelles et religieuses ainsi que les rôles de genre (Briggs et McBeath, 2010; Gallardo et Curry, 2009; Pumariega et Rothe, 2003; Pumariega et al., 2010). Cette prise en compte inclut de nommer les ressemblances et différences culturelles entre la culture d'accueil et la culture d'origine du jeune (Briggs et McBeath, 2010). L'intervenant doit donc être habilité à juger adéquatement ces paramètres ainsi que leur influence pour le jeune auprès duquel il intervient (Briggs et McBeath, 2010; Venable et Guada, 2014). L'intervention culturellement compétente auprès d'adolescents de minorités ethnoculturelles implique la considération des complexités liées à la biculturalité et la consolidation de l'identité ethnique, deux défis supplémentaires auxquels doivent faire face ces jeunes (Ashley et Brown, 2015; Huey Jr et Jones, 2013; Liddle, JacksonGilfort et Marvel, 2006). 
Immigration. Les auteurs recommandent de prendre en compte l'historique d'immigration et les possibles pertes, traumas ou abus qui y sont reliés dans l'évaluation de la problématique (Pumariega et al., 2013; Pumariega et al., 2010; Venable et Guada, 2014). Cela implique de poser ces questions de manière explicite au jeune et d'étudier son histoire de vie et ses premières expériences à la suite de l'immigration (Swearingen, 2002). Le niveau d'acculturation, la présence de stress d'acculturation et les conflits d'acculturation intrafamiliaux, particulièrement prévalents à l'adolescence, doivent aussi être explorés par l'intervenant afin de favoriser l'alliance (Pumariega et al., 2013; Pumariega et al., 2010).

Communication. Au-delà du soutien linguistique offert par le biais d'interprètes évoqué précédemment, il est important de respecter les moyens de communication privilégiés dans la famille du jeune (Pumariega et Rothe, 2003; Pumariega et al., 2010). Cela implique d'évaluer les thèmes, le langage et les expressions culturelles utilisées par le jeune (Briggs et McBeath, 2010; Pumariega et al., 2010). Plus précisément, cette évaluation doit inclure le langage relié aux émotions et aux cognitions qu'il utilise ainsi que les représentations qui en découlent (Swearingen, 2002). Ultimement, ces informations devraient permettre à l'intervenant d'utiliser des concepts culturellement pertinents pour le jeune (Gallardo et Curry, 2009). L'encouragement de l'apprentissage et l'utilisation de la langue d'origine à la maison favorisent également l'établissement et le maintien de l'alliance auprès d'adolescents de minorités ethnoculturelles en contexte d'intervention contrainte (Pumariega et al., 2010). De plus, malgré la valeur relative du respect de la confidentialité et du dévoilement de soi dans tout type d'intervention, ces deux éléments semblent particulièrement importants afin de favoriser le lien de confiance auprès des jeunes de minorités ethnoculturelles (Breland-Noble et al., 2010; Brown, Holloway, Akakpo et Aalsma, 2014; Gallardo et Curry, 2009; Henriksen, Degner et Oscarsson, 2008; Himelstein, 2011; Lee et al., 2006; Manso, Rauktis et Boyd, 2008; Pumariega et al., 2010; Scarborough, Taylor et Tuttle, 2013; Yoder et Ruch, 2015)

Difficultés et symptômes. II est important de comprendre la problématique du jeune par l'entremise des lentilles culturelles afin d'interpréter adéquatement son comportement, ce qui requiert généralement plus de temps que les pratiques courantes (Scarborough et al., 2013). L'évaluation doit prendre en compte ces variations, qui peuvent influencer l'expression et l'interprétation des affects, le degré de dévoilement (particulièrement lors de l'évaluation de la dangerosité de jeune pour lui-même ou pour les autres) et le développement de la symptomatologie (Pumariega et Rothe, 2003; Pumariega et al., 2013; Pumariega et al., 2010). Il est également important d'identifier, d'aborder et de freiner la portée des barrières à la demande d'aide, que celles-ci soient 
économiques, géographiques ou liées aux croyances culturelles (Bell, Wells et Merritt, 2009; Huey Jr et Jones, 2013). II faut donc éviter de stéréotyper la situation du jeune et de sa famille, mais tout de même la normaliser afin de réduire le stigma vécu (Breland-Noble et al., 2010; Briggs et McBeath, 2010; Lee et al., 2006; Pumariega et al., 2013; Pumariega et al., 2010). L'éducation sur le trouble ou les difficultés vécus et l'explication des différentes étapes de l'intervention peut aussi aider à réduire le stigma (Breland-Noble et al., 2010). II faut également comprendre et évaluer les mécanismes par lesquels le jeune et sa famille vont chercher du soutien, que ce soit au sein de la famille ou dans la communauté (p. ex., auprès d'amis, dans une institution religieuse) (Briggs et McBeath, 2010).

Buts et moyens. Les buts et moyens utilisés dans l'intervention doivent être congruents avec les valeurs et croyances culturelles du jeune (Briggs et McBeath, 2010; Gallardo et Curry, 2009). II faut donc ajuster la cible ou les modalités d'intervention en considérant ces valeurs, ce qui implique parfois une centration sur le problème ou les comportements problématiques, dans l'ici et le maintenant, et la proposition de stratégies comportementales en ligne avec ces valeurs (Brown et al., 2014; Cannon et Levy, 2008; Pumariega et Rothe, 2003; Pumariega et al., 2013; Pumariega et al., 2010). L'intervenant doit alors suivre le rythme du client avant d'aborder des thèmes plus intimes ou d'intervenir plus directement sur les affects (Brown et al., 2014; Karver et al., 2008; Ungar et lkeda, 2016). II est également bénéfique que l'intervenant soit spécifique quant aux buts et qu'il se positionne comme consultant afin d'aider le jeune dans ce que ce dernier considère lui-même le plus urgent (Gallardo et Curry, 2009).

\section{Compétences auprès des partenaires impliqués dans les milieux de vie du jeune}

Milieu familial. Plusieurs études pointent vers les bienfaits d'une intervention systémique qui favorise l'engagement de la famille en appui à l'intervention. Cette approche encourage les liens intrafamiliaux par le partage et l'accueil des points de vue de tous les membres de la famille des adolescents de minorités ethnoculturelles en contexte d'intervention contrainte (Bell et al., 2009; Briggs et McBeath, 2010; Byers et Lutz, 2015; Gallardo et Curry, 2009; Harvey et Hill, 2004; Pumariega et Rothe, 2003; Sharkey et al., 2010; Yoder et Ruch, 2015). Pour les jeunes de cultures traditionnelles, il est recommandé d'inclure dans l'intervention les membres de la famille élargie ou les amis proches de la famille (DeLoach, Dvorsky et White-Johnson, 2013; Gallardo et Curry, 2009; Pumariega et al., 2013; Pumariega et al., 2010; Scarborough et al., 2013; Venable et Guada, 2014). Il est également important de respecter les rôles établis culturellement dans la famille, ce qui peut vouloir dire de s'adresser d'abord et avant tout à la personne détenant l'autorité dans la famille (Scarborough et al., 2013). Centrer l'intervention dans la famille peut également être en accord avec les 
valeurs culturelles (Pumariega et al., 2010). Il est aussi important de donner du pouvoir d'agir au jeune et à sa famille dans les prises de décisions (Brown et al., 2014; Colle-Plamondon, 2014; DeLoach et al., 2013; Guay, 2010; Henriksen et al., 2008; Pumariega et Rothe, 2003; Pumariega et al., 2010; Scarborough et al., 2013; Venable et Guada, 2014; Yoder et Ruch, 2015), de prendre le temps d'offrir de l'information et des clarifications sur la problématique, les objectifs et les étapes de l'intervention (Breland-Noble et al., 2010; Cordaro,Tubman, Wagner et Morris, 2012; Pumariega et al., 2013; Sharkey et al., 2010) et de travailler à partir des forces (particulièrement celles liés à des valeurs culturelles) des jeunes et de leurs familles pour favoriser l'alliance (Cunningham, Foster et Warner, 2010; Harvey et Hill, 2004; Pumariega et al., 2013; Richardson, 2009; Sharkey et al., 2010).

Milieu d'intervention. Il est recommandé de mener l'intervention dans un environnement familier autant que possible, que cela soit à domicile ou en communauté, car les interventions en milieu institutionnel peuvent raviver des traumas d'oppression passés (Pumariega et al., 2013; Pumariega et al., 2010). Toutefois, lorsque l'hébergement en milieu institutionnel est nécessaire, il est favorable pour l'alliance que l'intervenant passe aussi du temps avec le jeune à l'extérieur du contexte institutionnel et qu'il soit flexible et disponible à le faire (Breland-Noble et al., 2010; Lee et al., 2006). II est également recommandé de favoriser les liens entre les jeunes qui reçoivent des services en unité (Colle-Plamondon, 2014).

Milieu communautaire. II est enfin recommandé aux intervenants d'encourager les familles à utiliser les ressources communautaires, tout en encourageant les partenariats entre ces dernières et les services d'intervention contraints (Bell et al., 2009; Pumariega et Rothe, 2003). Cela inclut la collaboration des ressources religieuses ou spirituelles et leurs principaux acteurs, tels que les prêtres (Breland-Noble, Belle et Burriss, 2011; Breland-Noble et al., 2010; Pumariega et Rothe, 2003; Pumariega et al., 2013). Les références vers des ressources de soutien matériel (dons de vêtements, de meubles, etc.) semblent également favoriser l'alliance (Guay, 2010).

\section{Savoir-être}

Biais culturels. Finalement, certains savoir-être favorisent l'alliance avec les adolescents de minorités ethnoculturelles recevant de l'aide contrainte. D'abord, l'intervenant doit être conscient et identifier ses propres biais culturels (DeLoach et al., 2013; Lindsey, 2010; Pumariega et al., 2013; Pumariega et al., 2010). L'intervenant peut faire cette tâche à l'aide des manifestations de son contre-transfert culturel, telles que la méfiance et la suspicion excessive à l'égard des adolescents ou de leur famille, le déni des différences culturelles ou une curiosité excessive pour la culture du jeune, curiosité pouvant s'apparenter à du tourisme culturel (Pumariega et al., 
2010). Peu de recommandations sont toutefois émises dans les écrits scientifiques sur les stratégies à adopter une fois les biais personnels identifiés, Byers et Lutz (2015) suggèrent de s'y attaquer par la restructuration cognitive.

Empathie. Le fait d'aborder ses biais culturels permet une meilleure compréhension du vécu du jeune. Le travail sur les biais de l'intervenant semble donc préalable à une réelle empathie au vécu du jeune, composante centrale à l'établissement de l'alliance thérapeutique, tout particulièrement en contexte d'intervention contrainte (Byers et Lutz, 2015; Karver et al., 2008; Venable et Guada, 2014; Yoder et Ruch, 2015). Celle-ci se caractérise par la capacité de l'intervenant à comprendre le vécu et les émotions du jeune ainsi qu'à prendre soin de ce dernier (Brown et al., 2014; Henriksen et al., 2008). Elle permet aussi une compréhension empathique de la fonction de protection de l'estime de soi, des résistances provenant des jeunes ou de leur famille, susceptibles de se manifester au cours de l'intervention (Guay, 2010). II semble toutefois important de démontrer cette compréhension du vécu du jeune par le biais de la congruence, de l'authenticité, de la chaleur et d'un regard positif inconditionnel (Brown et al., 2014; Byers et Lutz, 2015; Lindsey, 2010). Cette empathie se manifeste également par l'engagement dans la relation, et ce, même lorsque le jeune s'oppose ou manifeste des comportements perturbateurs (Manso et al., 2008).

Ouverture. À la suite du travail sur les biais personnels et l'empathie que ce travail permet de véhiculer au jeune, l'intervenant doit adopter une posture d'ouverture face au jeune. Cette posture se manifeste principalement dans le respect du point de vue du jeune et de sa famille, dans leurs mœurs, coutumes et valeurs (Guay, 2010; Lee et al., 2006). La démonstration de respect pour le jeune peut se traduire par la reconnaissance du besoin du jeune d'être reconnu pour qui il est, au-delà des motifs qui ont mené à l'intervention (Colle-Plamondon, 2014). Il semble également important de lui démontrer qu'il est pris au sérieux (Henriksen et al., 2008). Le respect du point de vue de la famille passe par la légitimation de ses connaissances sur leur propre enfant (Pumariega et al., 2010; Yoder et Ruch, 2015). Le respect du point de vue des mœurs et coutumes peut se manifester par l'acceptation de nourriture et de cadeaux, le maintien d'une distance appropriée, l'utilisation de salutations couramment utilisées, etc. (Pumariega et al., 2010; Scarborough et al., 2013). L'ouverture à travailler en fonction des valeurs, des croyances spirituelles et religieuses et des rôles de genre du jeune et de sa famille prend une place très importante dans la posture de savoir-être (Pumariega et al., 2010). Celle-ci implique que l'intervenant, en ayant fait un travail sur ses propres biais culturels et étant en mesure d'être empathique aux besoins des receveurs de services, soit capable de mettre de côté ses propres valeurs afin de négocier entre celles- 
ci, celles véhiculées par la loi et celles du jeune ou de sa famille (CohenÉmérique, 1993; Pumariega et al., 2010; Ungar et Ikeda, 2016).

\section{DISCUSSION}

La recension des différentes pratiques cliniques recommandées afin de favoriser l'alliance thérapeutique auprès d'adolescents de minorités ethnoculturelles en contexte d'intervention contrainte permet de confirmer la présence de telles recommandations dans les écrits scientifiques. La redondance des pratiques recommandées entre les différents milieux d'intervention contrainte inclus dans la recension (psychiatrie, protection de la jeunesse, justice pénale pour adolescents) suggère qu'il est possible de généraliser les recommandations d'un milieu à l'autre pour des jeunes ayant des profils similaires.

L'analyse des résultats pointe vers certains constats importants. La recommandation initiale sur le plan du savoir-être, c'est-à-dire l'identification de ses propres biais culturels à l'aide du contre-transfert culturel, semble être un élément précurseur central aux recommandations suivantes. $\mathrm{Ce}$ contre-transfert est obligatoire et nécessaire, tout intervenant portant un bagage culturel qu'il incarne (Rouchon, Reyre, Taïeb et Moro, 2009). L'action d'éluder la différence perçue serait donc un des obstacles les plus importants à l'amélioration des pratiques auprès des populations ethniques minoritaires. Toutefois, l'identification de ses biais est une tâche particulièrement difficile, car 1) la dimension culturelle fait souvent en sorte que ces biais sont partagés de manière consensuelle et implicite par les autres membres du groupe d'appartenance de l'intervenant; 2) les intervenants, n'ayant pour la plupart jamais travaillé à l'étranger, n'ont pas eu à se dégager de ce consensus afin d'observer leurs réactions qui, dans ce contexte, seraient vécues de manière beaucoup plus singulière (Rouchon et al., 2009). Les recommandations subséquentes sur le plan du savoir-être, se résumant en une capacité à être empathique et ouvert aux particularités des receveurs de services, constituent une posture à privilégier auprès de toute population minoritaire, que ce statut de minorité relève de la culture, de l'identité de genre, du revenu ou autre (Karver et al., 2008). Au-delà des populations minoritaires, l'individualité de chaque receveur de services met les habiletés d'adaptation à la différence de l'intervenant à l'épreuve, ce qui renvoie donc toujours aux mêmes savoirêtre sollicités par le travail auprès d'adolescents de minorités ethnoculturelles tels que recensés dans cet article, c'est-à-dire la prise de conscience et le travail sur les biais, l'empathie et l'ouverture. De ces attitudes découlent les savoir-faire recommandés dans les écrits, qui mettent essentiellement de l'avant l'importance de la prise en compte des différences culturelles à tous les niveaux de l'intervention. Bien qu'appliquées spécifiquement aux jeunes de minorités ethnoculturelles, 
certaines de ces pratiques ont déjà été documentées comme à privilégier pour un large éventail de populations, notamment la valorisation de l'alliance, de l'engagement et du pouvoir d'agir par la prise en compte des particularités du vécu des receveurs de services (Horvath et Symonds, 1991; Lemay, 2007). Ces recommandations sont en parfait accord avec les recommandations liées au savoir-être, appuyant ainsi le constat de la pertinence de prise en compte des différences pour toute population. Bien que les auteurs suggèrent d'enseigner les savoirs par le biais de formation, les différentes connaissances requises, comme celles liées à la culture et au contexte de vie, ne peuvent pas être généralisées à tout individu provenant d'une culture donnée. Au-delà de référents communs partagés par un groupe, la culture s'exprime individuellement: traiter de la culture d'un groupe minoritaire comme un tout homogène, univoque, vécu à l'identique par chacun des membres peut contribuer à alimenter les stéréotypes et le profilage, tout en limitant la prise en compte de l'individualité du receveur de services. Sur le plan pratique, il est aussi impossible de couvrir par une formation formelle l'ensemble des cultures des clients actuels et à venir. II semble plus pertinent d'explorer les contenus culturels directement auprès du receveur de services lui-même en mobilisant les savoir-être et savoir-faire exposés précédemment.

Ainsi, relativement au constat que les recommandations recensées pourraient s'appliquer à tout groupe de population qui inclut une certaine diversité, on peut en venir à questionner la pertinence même du concept de " compétence culturelle » qui semble, en limitant la portée de ses pratiques aux minorités ethnoculturelles, se soustraire à un éventail de groupes minoritaires beaucoup plus large. II serait fécond de lui substituer un concept plus inclusif afin de désigner la compétence à intervenir auprès d'un large éventail de groupes minoritaires, comme " compétence d'intervention en contexte d'altérité ». Par la prévalence et la multiplicité des groupes minoritaires ainsi que la nature subjective de la différence interindividuelle à l'intérieur même de ses différents groupes, les compétences d'intervention en contexte d'altérité gagneraient à faire partie du bagage de tout intervenant en relation d'aide. Dans le même ordre d'idée, l'identification des biais culturels de l'intervenant par ses réactions de contre-transfert culturel pourrait être élargie au contexte d'altérité, étant donné que ce type de réactions peut se manifester à la suite du contact à plusieurs autres groupes minoritaires. Le concept de contre-transfert culturel conserve donc sa pertinence, car même auprès de minorités au niveau de l'identité de genre, par exemple, il existe certaines conceptions qui découlent tout autant de visions partagées dans la culture qui résultent de dimensions historiques, socioculturelles et idéologiques et qui renvoient à un consensus collectif implicite de ce qu'est « l'autre étranger » (Rouchon et al., 2009). 
La recension met également en lumière une variété de problèmes méthodologiques qui viennent limiter la validité des recommandations recensées. Tout d'abord, plusieurs conclusions d'études menées auprès d'adultes de minorités ethnoculturelles sont généralisées à des adolescents sans explication ou justification particulière, comme si ce qui s'applique aux adultes s'applique aussi nécessairement aux adolescents (Briggs et McBeath, 2010; Pumariega et Rothe, 2003; Pumariega et al., 2013; Pumariega et al., 2010). Le même procédé est utilisé lors d'études menées auprès de familles de minorités ethnoculturelles, où les recommandations sont émises pour les familles, sans distinction entre les membres, comme si une bonne pratique auprès des parents l'est de même nécessairement auprès des enfants (Guay, 2010; Scarborough et al., 2013). Par conséquent, les recommandations recensées font très peu mention de pratiques à encourager spécifiquement auprès d'adolescents et les enjeux et défis spécifiques à ce stade de développement sont peu considérés. Notamment, les défis liés à la consolidation de l'identité, les enjeux de biculturalité et les conflits intergénérationnels d'acculturation semblent très superficiellement pris en compte dans les recommandations proposées par les auteurs consultés. Ces enjeux sont pourtant centraux dans l'adaptation de ces jeunes (Berry, 2005; Gonzales, 2014).

Le caractère involontaire de la participation des adolescents semble également peu ou pas du tout pris en compte dans les paramètres importants dans l'intervention pour favoriser l'alliance. Ceci s'explique sans doute par la nature volontaire à la participation des projets de recherche. Cependant, la prédominance d'études de cas et d'études qualitatives aurait pu permettre, au moins minimalement, une analyse rétrospective de l'influence de ce paramètre pour les jeunes, leurs familles ou les intervenants. Malheureusement, ce travail n'a pas été fait dans la grande majorité des études recensées.

La prédominance d'études de cas et d'études qualitatives, bien que justifiée par l'objet à l'étude, met également en lumière le peu de recherches qui documentent l'effet de ces pratiques sur l'établissement de l'alliance auprès de ce groupe de population. Les conclusions de plusieurs études sont questionnables, comme des résultats non significatifs rapportés comme significatifs (Harvey et Hill, 2004; Karver et al., 2008), des déductions ou suppositions arbitraires sans fondements (Ashley et Brown, 2015; Breland-Noble et al., 2011) et des résultats généralisés d'interventions menées auprès d'autres populations (Byers et Lutz, 2015; Orsi et al., 2010; Pumariega et Rothe, 2003; Pumariega et al., 2013; Pumariega et al., 2010). Bien que les fondements théoriques sur lesquels se basent les études semblent adéquats et que plusieurs succès dans la pratique clinique sont rapportés, la présence de failles méthodologiques importantes, couplée au peu d'études expérimentales et quasi 
expérimentales recensées, remet lourdement en question le fondement empirique de certaines recommandations suggérées.

\section{CONCLUSION}

En somme, les résultats recensés permettent de diriger la pratique des intervenants œuvrant auprès d'adolescents de minorités ethnoculturelles et mettent en évidence l'importance des savoir-être et des savoir-faire dans l'établissement et le maintien d'une bonne alliance thérapeutique avec ces adolescents en contexte d'intervention contrainte. La pertinence de la prise en compte du système de valeurs et des coutumes du jeune a mené à la proposition d'un cadre de référence embryonnaire qui pourrait être appliqué à tout groupe en contexte d'altérité, allant au-delà des pratiques s'appliquant exclusivement aux populations ethnoculturelles minoritaires. II y a néanmoins un grand besoin d'études afin de valider la pertinence des pratiques promues dans le présent article. On doit aussi approfondir l'influence de ces pratiques en tenant compte des particularités développementales de l'adolescence puisque les résultats actuels proviennent de la généralisation de recherches auprès d'autres populations.

La présente recension comporte certaines limites. L'absence d'écrits répondant directement à la question de recherche est sans doute explicable par la spécificité du groupe de population à l'étude. L'alternative retenue, c'est-à-dire une recension systématique en trois parties, augmente le risque que les résultats soient des généralisations de résultats obtenus auprès d'un autre groupe de population. De plus, la recension pourrait avoir omis certains articles pertinents étant donné 1) le large éventail de termes pertinents pouvant se rapporter à la question de recherche, surtout en regard à l'alliance thérapeutique et aux groupes de minorités ethnoculturelles; 2) la multiplicité des bases de données pertinentes au sujet et à leurs différents traitements des mots-clés; 3) le choix d'exclure tout article publié dans une langue autre que le français et l'anglais. Finalement, le fait que la plupart des études étaient de nature qualitative et l'analyse thématique qui a servi à organiser les résultats impliquent une part de subjectivité venant des auteurs.

\section{RÉFÉRENCES}

Anyon, Y. (2011). Reducing racial disparities and disproportionalities in the child welfare system: Policy perspectives about how to serve the best interests of African American youth. Children and Youth Services Review, 33(2), 242-253.

Ashley, W. et Brown, J. C. (2015). Attachment tHAIRapy: A culturally relevant treatment paradigm for African American foster youth. Journal of Black Studies, 46(6), 587-604. doi:10.1177/0021934715590406

Aubret, J. et Gilbert, P. (2003). L'évaluation des compétences. Bruxelles, Belgique : Éditions Mardaga.

Bell, C. C., Wells, S. J. et Merritt, L. M. (2009). Integrating cultural competency and empiricallybased practices in child welfare services: A model based on community psychiatry field 
principles of health. Children and Youth Services Review, 31(11), 1206-1213. doi:10.1016/j.childyouth.2009.08.011

Berry, J. W. (2005). Acculturation: Living successfully in two cultures. International Journal of Intercultural Relations, 29(6), 697-712.

Berry, J. W., Phinney, J. S., Sam, D. L. et Vedder, P. (2006). Immigrant youth: Acculturation, identity, and adaptation. Applied Psychology, 55(3), 303-332.

Breland-Noble, A. M., Bell, C. C. et Burriss, A. (2011). "Mama just won't accept this": Adult perspectives on engaging depressed African American teens in clinical research and treatment. Journal of Clinical Psychology in Medical Settings, 18(3), 225-234. doi:10.1007/s10880-011-9235-6

Breland-Noble, A. M., Burriss, A. et Poole, H. K. (2010). Engaging depressed African American adolescents in treatment: Lessons from the AAKOMA PROJECT. Journal of Clinical Psychology, 66(8), 868-879. doi:10.1002/jclp.20708

Briggs, H. E. et McBeath, B. (2010). Infusing culture into practice: Developing and implementing evidence-based mental health services for African American foster youth. Child Welfare, 89(1), 31-60.

Brown, J. R., Holloway, E. D., Akakpo, T. F. et Aalsma, M. C. (2014). "Straight up": Enhancing rapport and therapeutic alliance with previously-detained youth in the delivery of mental health services. Community Mental Health Journal, 50(2), 193-203. doi:10.1007/s10597013-9617-3

Byers, A. N. et Lutz, D. J. (2015). Therapeutic alliance with youth in residential care: challenges and recommendations. Residential Treatment for Children \& Youth, 32(1), 1-18.

Cahalane, H. (2013). Contemporary issues in child welfare practice. Dans Contemporary issues in child welfare practice (pp. xiv, 319). New York, NY: Springer.

Cannon, E. et Levy, M. (2008). Substance-using Hispanic youth and their families: Review of engagement and treatment strategies. The Family Journal, 16(3), 199-203. doi:10.1177/1066480708317496

Chand, A. (2000). The over-representation of black children in the child protection system: possible causes, consequences and solutions. Child \& Family Social Work, 5(1), 67-77.

Cohen-Émérique, M. (1993). L'approche interculturelle dans le processus d'aide. Santé mentale au Québec, 18(1), 71-91.

Colle-Plamondon, M. (2014). Le lien intervenant-jeune en unité de réadaptation : de la relation d'aide à l'alliance thérapeutique dans un contexte d'autorité (Mémoire de maîrise). Université de Montréal, Montréal.

Cordaro, M., Tubman, J. G., Wagner, E. F. et Morris, S. L. (2012). Treatment process predictors of program completion or dropout among minority adolescents enrolled in a brief motivational substance abuse intervention. Journal of Child \& Adolescent Substance Abuse, 21(1), 51-68. doi:http://dx.doi.org/10.1080/1067828X.2012.636697

Couture, D. et Dufour, S. (2013). Collaboration des minorités visibles avec les services de protection: soutien informel et stratégies d'intervention. Revue québécoise de psychologie, 34(2), 147-170.

Cunningham, P. B., Foster, S. L. et Warner, S. E. (2010). Culturally relevant family-based treatment for adolescent delinquency and substance abuse: understanding within-session processes. Journal of Clinical Psychology, 66(8), 830-846.

DeLoach, K. P., Dvorsky, M. et White-Johnson, R. L. (2013). Culturally competent engagement of African American youth and families in school mental health services. Dans C. S. Clauss-Ehlers, Z. N. Serpell et M. D. Weist (dir.), Handbook of culturally responsive school mental health: Advancing research, training, practice, and policy (p. 59-75). New York, NY: Springer.

Drake, B., Jolley, J. M., Lanier, P., Fluke, J., Barth, R. P. et Jonson-Reid, M. (2011). Racial bias in child protection? A comparison of competing explanations using national data. Pediatrics, 127(3), 471-478.

Dumbrill, G. C. (2006). Parental experience of child protection intervention: A qualitative study. Child Abuse \& Neglect, 30(1), 27-37.

Dumbrill, G. C. (2010). Power and child protection: The need for a child welfare service users' union or association. Australian Social Work, 63(2), 194-206. 
Eid, P., Turenne, M. et Magloire, J. (2011). Profilage racial et discrimination systémique des jeunes racisés : Rapport de la consultation sur le profilage racial et ses conséquences. Québec, QC : Commission des droits de la personne et des droits de la jeunesse.

Fluke, J. D., Harden, B. J., Jenkins, M. et Ruehrdanz, A. (2011). Research synthesis on child welfare: Disproportionality and disparities. Disparities and Disproportionality in child welfare: Analysis of the Research, 1.

Gallardo, M. E. et Curry, S. J. (2009). Shifting perspectives: culturally responsive interventions with latino substance abusers. Journal of Ethnicity in Substance Abuse, 8(3), 314-329.

Gendreau, G. (2001). Jeunes en difficulté et intervention psychoéducative. Montréal, QC : Sciences et culture.

Gladstone, J., Dumbrill, G., Leslie, B., Koster, A., Young, M. et Ismaila, A. (2014). Understanding worker-parent engagement in child protection casework. Children and Youth Services Review, 44, 56-64.

Gonzales, O. L. B. (2014). Ethnic identity formation and juvenile delinquency (Thèse de doctorat). Azusa Pacific University, Californie.

Guay, J. (2010). Les familles récalcitrantes en Protection de la jeunesse. Santé mentale au Québec, 35(2), 47-59. doi:10.7202/1000553ar

Harvey, A. R. et Hill, R. B. (2004). Africentric youth and family rites of passage program: Promoting resilience among at-risk African American youths. Social Work, 49(1), 65-74.

Hassan, G. et Rousseau, C. (2007). La protection des enfants: enjeux de l'intervention en contexte interculturel. Association pour la recherche interculturelle, 45, 37-50.

Henriksen, A., Degner, J. et Oscarsson, L. (2008). Youths in coercive residential care: attitudes towards key staff members' personal involvement, from a therapeutic alliance perspective. European Journal of Social Work, 11(2), 145-159.

Hill, R. B. (2006). Synthesis of research on disproportionality in child welfare: An update. Washington, DC: Casey-CSSP Alliance for Racial Equity in the Child Welfare System.

Himelstein, S. (2011). Engaging the moment with incarcerated youth: An existential-humanistic approach. The Humanistic Psychologist, 39(3), 206-221. doi:10.1080/08873267.2011.592436

Hines, A. M., Lemon, K., Wyatt, P. et Merdinger, J. (2004). Factors related to the disproportionate involvement of children of color in the child welfare system: A review and emerging themes. Children and Youth Services Review, 26(6), 507-527.

Horvath, A. O. et Symonds, B. D. (1991). Relation between working alliance and outcome in psychotherapy: A meta-analysis. Journal of Counseling Psychology, 38(2), 139-149.

Huey Jr, S. J. et Jones, E. O. (2013). Improving treatment engagement and psychotherapy outcomes for culturally diverse youth and families. Dans F. A. Paniagua et A.-M. Yamada (dir.), Handbook of multicultural mental health: Assessment and treatment of diverse populations ( $2^{\mathrm{e}}$ éd. p. 427-444). San Diego, CA: Elsevier.

Karver, M., Shirk, S., Handelsman, J. B., Fields, S., Crisp, H., Gudmundsen, G. et McMakin, D. (2008). Relationship processes in youth psychotherapy: Measuring alliance, alliancebuilding behaviors, and client involvement. Journal of Emotional and Behavioral Disorders, 16(1), 15-28.

Lavergne, C., Dufour, S. et Couture, D. (2014). Causes et solutions reliées à la disproportion des enfants issus des groupes racialisés dans le système de protection de la jeunesse: point de vue des intervenants. Alterstice-Revue Internationale de la Recherche Interculturelle, 4(1), 17-30.

Lee, B. R., Munson, M. R., Ware, N. C., Ollie, M. T., Scott Jr, L. D. et McMillen, J. C. (2006). Experiences of and attitudes toward mental health services among older youths in foster care. Psychiatric Services, 57(4), 487-492. doi:10.1176/appi.ps.57.4.487

Lemay, L. (2007). L'intervention en soutien à l'empowerment: du discours à la réalité. La question occultée du pouvoir entre acteurs au sein des pratiques d'aide. Nouvelles pratiques sociales, $20(1), 165-180$.

Lemay, L. (2013). Pratiques évaluatives et structuration du rapport parent-intervenant dans le champ du travail social en contexte de protection de la jeunesse: enjeux, défis et repères pour l'action. Dans E. Harper et H. Dorvil (dir.), Le travail social : Théories, méthodologies et pratiques (p. 313-338). Québec, Québec: Presses de l'Université du Québec. 
Liddle, H. A., Jackson-Gilfort, A. et Marvel, F. A. (2006). An empirically supported and culturally specific engagement and intervention strategy for African American adolescent males. American Journal of Orthopsychiatry, 76(2), 215-225. doi:10.1037/0002-9432.75.2.215

Lindsey, M. A. (2010). What are depressed African American adolescent males saying about mental health services and providers? Dans W. E. Johnson Jr (dir.), Social work with African American males: Health, mental health, and social policy (p. 161-178). New York, NY: Oxford University Press.

Malhamé, Y. (2010). Conflits intergénérationnels au sein de familles immigrantes: L'expérience d'une approche de médiation familiale et interculturelle dans un contexte de protection de la jeunesse (Essai de maîtrise). Université de Montréal, Montréal.

Manso, A., Rauktis, M. E. et Boyd, A. S. (2008). Youth expectations about therapeutic alliance in a residential setting. Residential Treatment for Children \& Youth, 25(1), 55-72.

Meyer, O., Zane, N. et Cho, Y. I. (2011). Understanding the psychological processes of the racial match effect in Asian Americans. Journal of Counseling Psychology, 58(3), 335-345.

Ministre de l'Industrie. (2013). Immigration et diversité ethnoculturelle au Canada. Récupéré de http://www12.statcan.gc.ca/nhs-enm/2011/as-sa/99-010-x/99-010-x2011001-fra.pdf

Orsi, M. M., Lafortune, D. et Brochu, S. (2010). Care and control: Working alliance among adolescents in authoritarian settings. Residential Treatment for Children \& Youth, 27(4), 277-303. doi:10.1080/0886571X.2010.520637

Phinney, J. S. (1989). Stages of ethnic identity development in minority group adolescents. The Journal of Early Adolescence, 9(1-2), 34-49.

Phinney, J. S., Romero, I., Nava, M. et Huang, D. (2001). The role of language, parents, and peers in ethnic identity among adolescents in immigrant families. Journal of Youth and Adolescence, 30(2), 135-153.

Pineau-Villeneuve, C. (2015). D'un système à l'autre: facteurs de risque d'incidence LSJPA chez les jeunes pris en charge en protection de la jeunesse en raison de troubles de comportement (Mémoire de maîtrise). Université de Montréal, Montréal.

Pumariega, A. J. et Rothe, E. (2003). Cultural considerations in child and adolescent psychiatric emergencies and crises. Child and Adolescent Psychiatric Clinics of North America, 12(4), 723-744. doi:10.1016/S1056-4993(03)00036-1

Pumariega, A. J., Rothe, E., Mian, A., Carlisle, L., Toppelberg, C., Harris, T.,... Smith, J. (2013). Practice parameter for cultural competence in child and adolescent psychiatric practice. Journal of the American Academy of Child \& Adolescent Psychiatry, 52(10), 1101-1115.

Pumariega, A. J., Rothe, E., Song, S. et Lu, F. G. (2010). Culturally informed child psychiatric practice. Child \& Adolescent Psychiatric Clinics of North America, 19(4), 739-757. doi:10.1016/j.chc.2010.07.004

Renou, M. (2005). Psychoéducation : Une conception, une méthode. Montréal, QC: Sciences et Cultures.

Richardson, B. (2009). Comparative analysis of two community-based efforts designed to impact disproportionality. Child Welfare, 87(2), 297-317.

Rouchon, J.-F., Reyre, A., Taïeb, O. et Moro, M. R. (2009). L'utilisation de la notion de contretransfert culturel en clinique. L'Autre, 10(1), 80-89.

Scarborough, N., Taylor, B. et Tuttle, A. (2013). Collaborative home-based therapy (CHBT): A culturally responsive model for treating children and adolescents involved in child protective service systems. Contemporary Family Therapy, 35(3), 465-477.

Sharkey, J. D., Sander, J. B. et Jimerson, S. R. (2010). Acculturation and mental health: Response to a culturally-centered delinquency intervention. Journal of Criminal Justice, 38(4), 827-834. doi:10.1016/j.jcrimjus.2010.05.011

Sinclair, M. M. et Smith, B. D. (2016). Engaging urban African American adolescents in treatment. Dans R. Wells-Wilbon, A. R. McPhatter et H. F. O. Vakalahi (dir.), Social work practice with African Americans in urban environments (p.55-76). New York, NY: Springer.

Soriano, F. I., Rivera, L. M., Williams, K. J., Daley, S. P. et Reznik, V. M. (2004). Navigating between cultures: The role of culture in youth violence. Journal of Adolescent Health, 34(3), 169-176.

Swearingen, L. A. (2002). A grounded theory of clinical engagement with juvenile offenders (Thèse de doctorat). University of Pittsburgh, Pittsburgh. 
Torres, L. et Ong, A. D. (2010). A daily diary investigation of latino ethnic identity, discrimination, and depression. Cultural Diversity and Ethnic Minority Psychology, 16(4), 561-568.

Ungar, M. et Ikeda, J. (2016). Rules or no rules? Three strategies for engagement with young people in mandated services. Child and Adolescent Social Work Journal, 34(3), 259-267. doi:10.1007/s10560-016-0456-2

Venable, V. M. et Guada, J. (2014). Culturally Competent Practice with African American juvenile sex offenders. Journal of Child Sexual Abuse, 23(3), 229-246. doi:10.1080/10538712.2014.888122

Yoder, J. et Ruch, D. (2015). Youth who have sexually offended: Using strengths and rapport to engage families in treatment. Journal of Child and Family Studies, 24(9), 2521-2531. doi:10.1007/s10826-014-0054-x

\section{RÉSUMÉ}

Le but de cette recension est de faire le point sur les pratiques cliniques recommandées afin de favoriser l'alliance thérapeutique dans l'intervention auprès d'adolescents de minorités ethnoculturelles dans des contextes d'intervention contrainte (protection de la jeunesse, justice pénale pour adolescents, psychiatrie). L'analyse des résultats des 32 articles sélectionnés permet de répertorier des pratiques s'organisant autour du savoir, du savoir-être et du savoirfaire. Cette analyse met également en lumière des biais méthodologiques importants dans les recherches entourant le sujet et une absence de soutien empirique des pratiques. Des orientations pour la pratique clinique et les recherches futures sont proposées.

\section{MOTS-CLÉS}

alliance thérapeutique, adolescents, minorité ethnoculturelle, intervention contrainte

\section{ABSTRACT}

The aim of this review is to sum up the recommended clinical practices that promote therapeutic alliance in interventions conducted with ethnocultural minority adolescents in mandatory intervention settings (child welfare, juvenile justice, psychiatry). The analysis of the results stemming from the 32 selected articles allows to list practices that can be organised around knowledge, dispositions and skills. The analysis also brings to light important methodological biases in studies on the topic, as well as an absence of empirical support for the practices. Directions for clinical practice and future research are proposed.

\section{KEYWORDS}

therapeutic alliance, adolescents, ethnocultural minority, mandatory intervention 
Tableau 1

Recension 1 : Pratiques culturellement compétentes auprès d'adolescents en contexte d'intervention contrainte

\begin{tabular}{|c|c|c|c|c|}
\hline Études & Échantillon & Milieu* & Devis & But(s) \\
\hline $\begin{array}{l}\text { Ashley et } \\
\text { Brown (2015) }\end{array}$ & $\begin{array}{l}9 \text { filles afro-américaines âgées de } 10 \\
\text { à } 18 \text { ans en famille d'accueil }\end{array}$ & PJ & Qualitatif & $\begin{array}{l}\text { Évaluer les bienfaits de l'« Attachement tHAIRapy », un pairage } \\
\text { entre coiffure et psychothérapie }\end{array}$ \\
\hline $\begin{array}{l}\text { Bell, Wells et } \\
\text { Merritt (2009) }\end{array}$ & - & PJ & Théorique & $\begin{array}{l}\text { Adapter un modèle d'intervention culturellement compétent à } \\
\text { l'intervention en protection de la jeunesse }\end{array}$ \\
\hline $\begin{array}{l}\text { Briggs et } \\
\text { McBeath } \\
\text { (2010) }\end{array}$ & - & PJ & Théorique & $\begin{array}{l}\text { Compte-rendu des conséquences du manque d'accès à des } \\
\text { interventions culturellement compétentes pour les jeunes afro- } \\
\text { américains en famille d'accueil. Offrir un cadre pour développer } \\
\text { ce type d'intervention. }\end{array}$ \\
\hline $\begin{array}{l}\text { Cunningham, } \\
\text { Foster et } \\
\text { Warner (2010) }\end{array}$ & $\begin{array}{l}\text { Adolescents blancs ou afro- } \\
\text { américains ayant des troubles de } \\
\text { comportements et leurs parents }\end{array}$ & JPA & Mixte & $\begin{array}{l}\text { Décrire le développement d'un traitement culturellement } \\
\text { compétent pour des familles afro-américaines anglophones à } \\
\text { partir du « Multisystemic Therapy » }\end{array}$ \\
\hline $\begin{array}{l}\text { Harvey et Hill } \\
(2004)\end{array}$ & $\begin{array}{l}\text { Garçons afro-américains âgés de } 11 \\
\text { à } 14 \text { ans qui sont en relation avec le } \\
\text { système de JPA }\end{array}$ & JPA & $\begin{array}{l}\text { Quasi- } \\
\text { expérimental }\end{array}$ & $\begin{array}{l}\text { Examiner l'effet d'une intervention afrocentrique sur des } \\
\text { adolescents à risque («MAAT Adolescent » et « Family Rites of } \\
\text { Passage ») }\end{array}$ \\
\hline $\begin{array}{l}\text { Pumariega et } \\
\text { Rothe (2003) }\end{array}$ & $\begin{array}{l}\text { Familles de minorités } \\
\text { ethnoculturelles }\end{array}$ & PSY & $\begin{array}{l}\text { Théorique / } \\
\text { Étude de cas }\end{array}$ & $\begin{array}{l}\text { Examiner les éléments à considérer lors d'interventions } \\
\text { d'urgences auprès d'une population de minorités ethnoculturelles }\end{array}$ \\
\hline $\begin{array}{l}\text { Pumariega et } \\
\text { al. (2013) }\end{array}$ & $\begin{array}{l}\text { Enfants et adolescents de minorités } \\
\text { ethnoculturelles }\end{array}$ & PSY & $\begin{array}{l}\text { Revue } \\
\text { systématique }\end{array}$ & $\begin{array}{l}\text { Revue systématique des pratiques culturellement compétentes } \\
\text { auprès d'enfants et d'adolescents de minorités ethnoculturelles }\end{array}$ \\
\hline $\begin{array}{l}\text { Pumariega, } \\
\text { Rothe, Song et } \\
\text { Lu (2010) }\end{array}$ & $\begin{array}{l}\text { Enfants et adolescents de minorités } \\
\text { ethnoculturelles }\end{array}$ & PSY & Théorique & $\begin{array}{l}\text { Identifier les défis et recommandations pour des interventions } \\
\text { culturellement compétentes auprès d'enfants et d'adolescents de } \\
\text { minorités ethnoculturelles }\end{array}$ \\
\hline
\end{tabular}


Tableau 1

Recension 1 : Pratiques culturellement compétentes auprès d'adolescents en contexte d'intervention contrainte (suite)

\begin{tabular}{|l|l|l|l|l|}
\hline Études & Échantillon & Milieu* & Devis & But(s) \\
\hline $\begin{array}{l}\text { Richardson } \\
(2009)\end{array}$ & $\begin{array}{l}\text { Familles afro-américaines et } \\
\text { autochtones }\end{array}$ & PJ & Mixte & $\begin{array}{l}\text { Connaître les effets d'une intervention visant à réduire la } \\
\text { surreprésentation des jeunes de minorités ethnoculturelles et } \\
\text { autochtones dans les services de protection }\end{array}$ \\
\hline $\begin{array}{l}\text { Scarborough, } \\
\text { Taylor etTuttle } \\
(2013)\end{array}$ & $\begin{array}{l}\text { Enfants et adolescents de minorités } \\
\text { ethnoculturelles }\end{array}$ & PJ & Théorique & $\begin{array}{l}\text { Présentation d'un modèle d'intervention culturellement compétent } \\
\text { auprès d'enfants et d'adolescents dans les services de protection }\end{array}$ \\
\hline $\begin{array}{l}\text { Sharkey, } \\
\text { Sander } \\
\text { etJimerson } \\
(2010)\end{array}$ & $\begin{array}{l}\text { Groupes d'adolescents en probation } \\
\text { âgés de 15 à 17 ans. 68 \% de } \\
\text { garçons, 32\% de filles. Groupe de } \\
\text { prédominance latino-américaine. }\end{array}$ & JPA & $\begin{array}{l}\text { Quasi- } \\
\text { expérimental }\end{array}$ & $\begin{array}{l}\text { Évaluer l'efficacité d'une intervention culturellement centrée dans } \\
\text { la réduction de symptômes internalisés et externalisés }\end{array}$ \\
\hline $\begin{array}{l}\text { Venable et } \\
\text { Guada (2014) }\end{array}$ & $\begin{array}{l}\text { Adolescents afro-américains ayant } \\
\text { commis un délit sexuel }\end{array}$ & JPA & $\begin{array}{l}\text { Théorique / } \\
\text { Étude de cas }\end{array}$ & $\begin{array}{l}\text { Identifier les défis et recommandations pour des interventions } \\
\text { culturellement compétentes auprès de cette population }\end{array}$ \\
\hline
\end{tabular}


Tableau 2

Recension 2 : Pratiques favorisant l'alliance thérapeutique auprès d'adolescents en contexte d'intervention contrainte

\begin{tabular}{|c|c|c|c|c|}
\hline Études & Échantillon & Milieu & Devis & But(s) \\
\hline $\begin{array}{l}\text { Brown, } \\
\text { Holloway, } \\
\text { Akakpo et } \\
\text { Aalsma (2014) }\end{array}$ & $\begin{array}{l}\text { Jeunes âgés de } 11 \text { à } 17 \text { ans, } \\
\text { ayant été incarcérés. } 12 \\
\text { garçons et } 7 \text { filles }\end{array}$ & JPA & $\begin{array}{l}\text { Qualitatif } \\
\text { (Théorisation } \\
\text { ancrée) }\end{array}$ & $\begin{array}{l}\text { Comprendre comment des jeunes ayant été incarcérés ont vécu } \\
\text { l'alliance thérapeutique avec leur intervenant }\end{array}$ \\
\hline $\begin{array}{l}\text { Byers et Lutz } \\
(2015)\end{array}$ & Jeunes en hébergement & PJ & Théorique & $\begin{array}{l}\text { Identifier les défis, facilitateurs et obstacles au développement de } \\
\text { l'alliance thérapeutique }\end{array}$ \\
\hline $\begin{array}{l}\text { Colle- } \\
\text { Plamondon } \\
(2014)\end{array}$ & $\begin{array}{l}7 \text { intervenants et } 6 \text { jeunes âgés } \\
\text { de } 15 \text { à } 17 \text { ans en unité de } \\
\text { réadaptation en CJ }\end{array}$ & PJ & $\begin{array}{l}\text { Qualitatif } \\
\text { (Théorisation } \\
\text { ancrée) }\end{array}$ & Documenter la relation d'aide en contexte d'autorité \\
\hline Guay (2010) & $\begin{array}{l}\text { Familles en protection de la } \\
\text { jeunesse }\end{array}$ & PJ & Théorique & $\begin{array}{l}\text { Aborder les défis cliniques auxquels les thérapeutes familiaux sont } \\
\text { confrontés en Centre Jeunesse }\end{array}$ \\
\hline $\begin{array}{l}\text { Henriksen, } \\
\text { Degner et } \\
\text { Oscarsson } \\
(2008)\end{array}$ & 46 jeunes de 14 à 20 ans & PJ & Qualitatif & $\begin{array}{l}\text { Explorer les obstacles et les opportunités, du point de vue des jeunes, à } \\
\text { l'établissement d'une alliance thérapeutique fructueuse avec leur } \\
\text { intervenant }\end{array}$ \\
\hline $\begin{array}{l}\text { Himelstein } \\
(2011)\end{array}$ & Adolescents en justice pénale & JPA & $\begin{array}{l}\text { Théorique / } \\
\text { Étude de cas }\end{array}$ & $\begin{array}{l}\text { Décrire une alternative à la thérapie traditionnelle avec les jeunes } \\
\text { incarcérés }\end{array}$ \\
\hline $\begin{array}{l}\text { Karver et al. } \\
(2008)\end{array}$ & $\begin{array}{l}23 \text { adolescents avec } \\
\text { symptômes dépressifs ayant } \\
\text { commis une tentative de } \\
\text { suicide. Majoritairement des } \\
\text { filles }\end{array}$ & PSY & $\begin{array}{l}\text { Quasi- } \\
\text { Expérimental }\end{array}$ & $\begin{array}{l}\text { Étudier les associations entre les construits de l'alliance dans une } \\
\text { comparaison entre un traitement cognitivo-comportemental et la } \\
\text { « Nondirective supportive therapy » }\end{array}$ \\
\hline
\end{tabular}


Tableau 2

Recension 2 : Pratiques favorisant l'alliance thérapeutique auprès d'adolescents en contexte d'intervention contrainte (suite)

\begin{tabular}{|c|c|c|c|c|}
\hline Études & Échantillon & Milieu & Devis & But(s) \\
\hline $\begin{array}{l}\text { Lee et al. } \\
(2006)\end{array}$ & 389 jeunes de 17 ans & PJ & Qualitatif & $\begin{array}{l}\text { Décrire les caractéristiques valorisées par les jeunes dans leur relation } \\
\text { avec leurs intervenants }\end{array}$ \\
\hline $\begin{array}{l}\text { Manso, } \\
\text { Rauktis et } \\
\text { Boyd (2008) }\end{array}$ & $\begin{array}{l}11 \text { garçons âgés de } 9 \text { à } 18 \text { ans } \\
\text { (14 ans en moyenne) en } \\
\text { hébergement }\end{array}$ & $\begin{array}{l}\text { PJ } / \\
\text { JPA }\end{array}$ & Qualitatif & $\begin{array}{l}\text { Mieux comprendre comment les jeunes en hébergement perçoivent leur } \\
\text { relation avec les intervenants, ce qu'ils considerent comme étant des } \\
\text { qualités importantes pour établir et maintenir la relation, et ceux qu'ils } \\
\text { considèrent comme des acteurs responsables de cette alliance }\end{array}$ \\
\hline $\begin{array}{l}\text { Orsi, Lafortune } \\
\text { et Brochu } \\
\text { (2010) }\end{array}$ & Adolescents et jeunes adultes & $\begin{array}{l}\text { PJ } \\
\text { IJPA / } \\
\text { PSY }\end{array}$ & $\begin{array}{l}\text { Revue } \\
\text { systématique }\end{array}$ & $\begin{array}{l}\text { Effectuer une recension des écrits concernant l'alliance thérapeutique } \\
\text { auprès d'adolescents et de jeunes adultes qui participent non } \\
\text { volontairement à un programme d'intervention }\end{array}$ \\
\hline $\begin{array}{l}\text { Swearingen } \\
(2002)\end{array}$ & $\begin{array}{l}\text { Adolescents de } 11 \text { à } 18 \text { ans } \\
\text { ayant un diagnostic de troubles } \\
\text { des conduites ou étant dans le } \\
\text { système de JPA }\end{array}$ & JPA & $\begin{array}{l}\text { Qualitatif } \\
\text { (Théorisation } \\
\text { ancrée) }\end{array}$ & $\begin{array}{l}\text { Générer une théorie de l'engagement clinique chez les adolescents } \\
\text { délinquants }\end{array}$ \\
\hline $\begin{array}{l}\text { Ungar et lkeda } \\
\text { (2016) }\end{array}$ & $\begin{array}{l}61 \text { jeunes en contexte } \\
\text { d'intervention contrainte }\end{array}$ & $\begin{array}{l}\text { PJ } / \\
\text { JPA } / \\
\text { PSY }\end{array}$ & $\begin{array}{l}\text { Qualitatif } \\
\text { (Théorisation } \\
\text { ancrée) }\end{array}$ & $\begin{array}{l}\text { Déterminer quel style relationnel des intervenants (permissif, autoritaire } \\
\text { ou démocratique) fonctionne le mieux avec quel type de jeunes, étudier } \\
\text { l'influence du milieu d'intervention sur la pertinence des différents styles } \\
\text { et obtenir le point de vue des jeunes sur ces questions }\end{array}$ \\
\hline $\begin{array}{l}\text { Yoder et Ruch } \\
\text { (2015) }\end{array}$ & 11 intervenants & JPA & Qualitatif & $\begin{array}{l}\text { Déterminer comment les familles de jeunes délinquants sexuels } \\
\text { deviennent engagées dans le traitement }\end{array}$ \\
\hline
\end{tabular}


Tableau 3

Recension 3 : Pratiques culturellement compétentes afin de favoriser l'alliance thérapeutique auprès des adolescents

\begin{tabular}{|c|c|c|c|}
\hline Études & Échantillon & Devis & But(s) \\
\hline $\begin{array}{l}\text { Breland- } \\
\text { Noble, Bell et } \\
\text { Burriss } \\
(2011)\end{array}$ & $\begin{array}{l}24 \text { parents afro-américains } \\
\text { (dont } 21 \text { femmes) }\end{array}$ & $\begin{array}{l}\text { Qualitatif } \\
\text { (Théorisation } \\
\text { ancrée) }\end{array}$ & $\begin{array}{l}\text { Identifier les facteurs que les adultes afro-américains considèrent importants dans } \\
\text { l'engagement au traitement d'adolescents dépressifs pour lesquels ils sont des } \\
\text { adultes significatifs }\end{array}$ \\
\hline $\begin{array}{l}\text { Breland- } \\
\text { Noble, } \\
\text { Burriss et } \\
\text { Poole (2010) }\end{array}$ & $\begin{array}{l}28 \text { adolescents afro- } \\
\text { américains âgés de } 11 \text { à } 17 \\
\text { ans souffrant d'une } \\
\text { dépression }\end{array}$ & $\begin{array}{l}\text { Qualitatif } \\
\text { (Théorisation } \\
\text { ancrée) }\end{array}$ & $\begin{array}{l}\text { Comprendre l'expérience d'adolescents afro-américains et trouver des manières } \\
\text { culturellement pertinentes pour les engager dans le traitement }\end{array}$ \\
\hline $\begin{array}{l}\text { Cannon and } \\
\text { Levy (2008) }\end{array}$ & $\begin{array}{l}\text { Jeunes hispanophones } \\
\text { consommateurs et leurs } \\
\text { familles }\end{array}$ & Théorique & $\begin{array}{l}\text { Recenser des études qui abordent l'engagement et le traitement de cette } \\
\text { population et mettre en lumière deux modèles théoriques prometteurs }\end{array}$ \\
\hline $\begin{array}{l}\text { Cordaro, } \\
\text { Tubman, } \\
\text { Wagner et } \\
\text { Morris (2012) }\end{array}$ & $\begin{array}{l}48 \text { Adolescents latino- } \\
\text { américains de } 14 \text { à } 19 \text { ans }\end{array}$ & Corrélationnel & $\begin{array}{l}\text { Déterminer si des variables spécifiques telles que l'alliance thérapeutique et } \\
\text { l'engagement prédisent ceux qui quittent et ceux qui terminent le traitement }\end{array}$ \\
\hline $\begin{array}{l}\text { DeLoach, } \\
\text { Dvorsky et } \\
\text { White- } \\
\text { Johnson } \\
(2013)\end{array}$ & $\begin{array}{l}\text { Jeunes afro-américains dans } \\
\text { les services de santé mentale } \\
\text { scolaire }\end{array}$ & $\begin{array}{l}\text { Recension } \\
\text { (Chapitre de } \\
\text { livre) }\end{array}$ & $\begin{array}{l}\text { Recenser les pratiques culturellement compétentes à l'engagement de jeunes afro- } \\
\text { américains et leurs familles dans les services de santé mentale scolaire }\end{array}$ \\
\hline $\begin{array}{l}\text { Gallardo et } \\
\text { Curry (2009) }\end{array}$ & $\begin{array}{l}\text { Adolescents latino- } \\
\text { américains avec des } \\
\text { problèmes de consommation }\end{array}$ & Théorique & $\begin{array}{l}\text { Mettre en lumière les étapes d'une adaptation d'un modèle thérapeutique basé sur } \\
\text { les forces }\end{array}$ \\
\hline
\end{tabular}


Tableau 3

Recension 3 : Pratiques culturellement compétentes afin de favoriser l'alliance thérapeutique auprès des adolescents (suite)

\begin{tabular}{|c|c|c|c|}
\hline $\begin{array}{l}\text { Huey Jr et } \\
\text { Jones (2013) }\end{array}$ & $\begin{array}{l}\text { Jeunes de minorités } \\
\text { ethnoculturelles }\end{array}$ & $\begin{array}{l}\text { Recension } \\
\text { systématique }\end{array}$ & $\begin{array}{l}\text { Répondre à cinq questions: Quelles approches augmentent l'engagement des } \\
\text { jeunes de minorités ethnoculturelles? Quelles interventions psychosociales sont } \\
\text { efficaces pour les jeunes de minorités ethnoculturelles? À quels points les effets de } \\
\text { ces traitements sont-ils robustes? Est-ce que les effets du traitement varient en } \\
\text { fonction du groupe ethnoculturel? Est-ce que l'adaptation à la culture augmente } \\
\text { l'efficacité des traitements? }\end{array}$ \\
\hline $\begin{array}{l}\text { Liddle, } \\
\text { Jackson- } \\
\text { Gilfort et } \\
\text { Marvel } \\
(2006)\end{array}$ & $\begin{array}{l}\text { Garçons afro-américains } \\
\text { antisociaux impliqués dans la } \\
\text { rue }\end{array}$ & Théorique & $\begin{array}{l}\text { Nommer les principales caractéristiques du « Multidimensional Family Therapy » } \\
\text { pour un groupe d'adolescents afro-américains antisociaux }\end{array}$ \\
\hline $\begin{array}{l}\text { Lindsey } \\
(2010)\end{array}$ & $\begin{array}{l}18 \text { garçons adolescents afro- } \\
\text { américains déprimés âgés de } \\
14 \text { à } 18 \text { ans }\end{array}$ & Qualitatif & $\begin{array}{l}\text { Explorer la perception des services de santé mentale et des intervenants chez des } \\
\text { jeunes garçons afro-américains déprimés concernant les facteurs qui influencent } \\
\text { l'engagement et l'alliance thérapeutique }\end{array}$ \\
\hline $\begin{array}{l}\text { Meyer, Zane } \\
\text { et Cho } \\
\text { (2011) }\end{array}$ & $\begin{array}{l}171 \text { filles âgées de } 17 \text { à } 24 \\
\text { ans (majoritairement } \\
\text { asiatiques) }\end{array}$ & Expérimental & $\begin{array}{l}\text { Tester l'effet du jumelage racial sur la crédibilité des thérapeutes et l'alliance. } \\
\text { Déterminer si cet effet et médiatisé par la perception de similarité et la perception } \\
\text { de soutien. Tester l'identité ethnique comme modérateur de la relation entre le } \\
\text { jumelage racial et les variables de processus. }\end{array}$ \\
\hline $\begin{array}{l}\text { Sinclair et } \\
\text { Smith (2016) }\end{array}$ & Adolescents afro-américains & $\begin{array}{l}\text { Théorique } \\
\text { (Chapitre de } \\
\text { livre) }\end{array}$ & $\begin{array}{l}\text { Examiner les stratégies culturellement pertinentes afin de favoriser l'engagement } \\
\text { d'adolescents afro-américains aux traitements }\end{array}$ \\
\hline
\end{tabular}

* Légende des milieux : PJ = Protection de la jeunesse; JPA = Justice pénale pour adolescents; PSY = Psychiatrie 


\section{Appendice}

Mots-clés utilisés pour les trois sous-recensions

\begin{tabular}{|c|c|}
\hline Recension 1 & 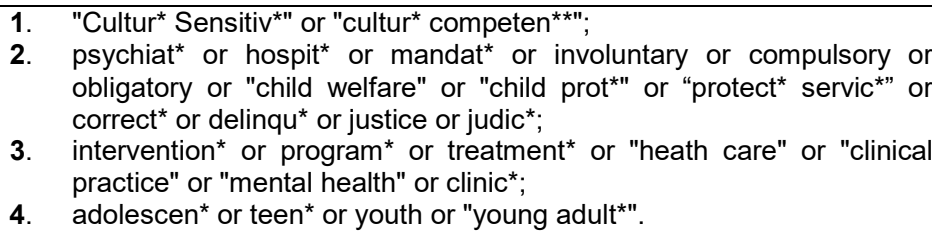 \\
\hline Recension 2 & 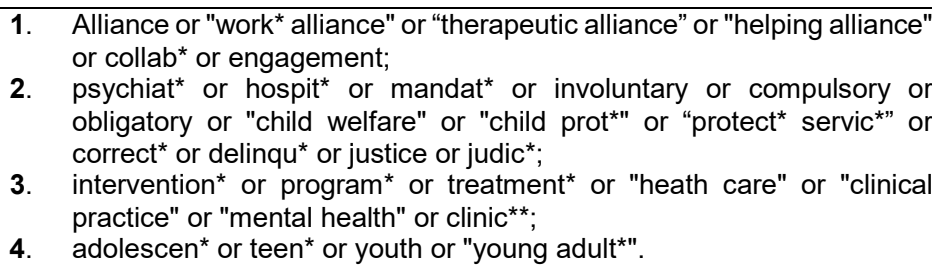 \\
\hline Recension 3 & $\begin{array}{l}\text { 1. } \text { minorit }^{*} \text { or rac } \text { ram }^{*} \text { or ethnic }{ }^{*} \text { or culture* or "Cultur* Sensitivity" or "culture* } \\
\text { 2. Alliance or "work* alliance" or "therapeutic alliance" or "helping alliance" } \\
\text { 2. or collab* or engagement; } \\
\text { 3. intervention* or program" or treatment* or "heath care" or "clinical } \\
\text { practice" or "mental health" or clinic*; } \\
\text { 4. adolescen* or teen* or youth or "young adult". }\end{array}$ \\
\hline
\end{tabular}

